\title{
Morphological processing of multichannel images
}

\author{
BALVINDER SINGH and M U SIDDIQI \\ Department of Electrical Engineering, Indian Institute of \\ Technology, Kanpur 208016, India
}

\begin{abstract}
A theory for morphological processing of multichannel images within the abstract framework of lattice theory is presented. The theory makes use of marginal ordering and reduced ordering schemes to arrive at multivariate morphological operators. This paper elucidates the algebraic structure and properties of mathematical multivariate morphology. It is shown that matrix morphology formulation is a natural consequence of marginal ordering and serves as a technique for processing of multichannel images. Further, the concepts of quasi-ordering and complete quasi-lattices are introduced to define morphological operators utilizing the reduced ordering scheme.
\end{abstract}

Keywords. Multichannel images; mathematical multivariate morphology; multivariate ordering; lattice theory; matrix operators; operators over quasilattices.

\section{Introduction}

Mathematical morphology is a theory which is concerned with the processing and analysis of topological and geometrical aspects of an image. It initially originated from a set theoretical formulation for processing of binary images (Matheron 1975; Serra 1982). Subsequently, these methods were extended for processing of gray scale images (Sternberg 1986). However, it was soon realized by Serra (1988), Ronse (1990), Heijmans \& Ronse (1990) and Ronse \& Heijmans (1991) that mathematical morphology can be generalized within the abstract framework of complete lattices. An excellent treatment of the recent developments in mathematical morphology is available in the text by Heijmans (1994).

There are many varied and important applications such as processing of colour images, multispectral image analysis, biomedical imaging, robot vision, industrial inspection etc. which require multichannel image processing. In these applications, the use of image data from multiple frequency bands, multiple time frames, multiple colours or multiple sensors (e.g. optical, radar, range etc.) is of tremendous use. For such situations, the information is available in the form of multivariate data which should be processed so as to take into account the interrelationship between the individual variates (image frames). A formulation based on 3-dimensional geometrical structure of image sequences is presented by Cheng \& Venetsanopoulos (1992). Another attempt has been to decorrelate the various signal 
components by means of a suitable linear transformation and then apply the morphological operators separately (Eo 1992).

We approach this problem by extending univariate morphology to multivariate morphology by the use of ordering principles for multivariate data. Several multivariate ordering techniques have been proposed and discussed (Barnett 1976). However, only marginal ordering and reduced ordering schemes are of interest to us. In marginal ordering, all components of the multivariate data are ordered componentwise. On the other hand, in reduced ordering, the multivariate data are mapped to completely ordered univariate data by using some distance criteria and then the ordering is performed on the basis of these univariate data. These ordering principles have been applied to develop nonlinear multivariate order statistic and vector median filters (Astola et al 1990; Hardie \& Arce 1991; Pitas \& Tsakalides 1991; Trahanias \& Venetsanopoulos 1993). During the preparation stages of this paper, the authors became aware of the work by Goutisias et al (1994). Their work deals with a lattice theory approach to multichannel morphological image processing (termed vector morphology by Goutisias et al (1994)) and consists of examples demonstrating its applications. This paper presents parallel theoretical results and supplements the work of Goutisias et al (1994). Due to limited space, we will not discuss any of the background theory and no proofs of the results presented will be given. However for an overview, readers may refer to details of lattice theory in texts by Birkhoff (1973), Fuchs (1963) and Gierz et al (1980) and mathematical morphology in texts by Heijmans (1994) and Serra $(1982,1988)$. Readers interested in the details of the work presented here may refer to Singh \& Siddiqi (1994).

This paper is organized as follows. Section 2 gives a rigorous treatment to multivariate morphology over general complete lattices within the marginal ordering scheme. Section 3 deals with translation invariant multivariate morphological operators and their properties. Section 4 addresses the problem of morphological operators utilizing the reduced ordering techniques. The conclusions are presented in $\$ 5$.

\section{Multivariate morphological operators}

\subsection{Structure of multivariate signal}

Let us consider a multichannel image (which is a multivariate signal) that is specified by an indexed set of image frames. Let $\mathcal{I}$ represent such an indexing set. For the discussion to follow, we have the set $\mathcal{I}=\{1,2, \ldots, m\}$. The range set for the $i$ th image channel is denoted by $\mathcal{G}_{i}$ for $i \in \mathcal{I}$. Then the cartesian product set

$$
\begin{aligned}
\mathcal{G} & =\prod_{i \in \mathcal{I}} \mathcal{G}_{i}=\mathcal{G}_{1} \times \mathcal{G}_{2} \times \cdots \times \mathcal{G}_{m} \\
& =\left\{G=\left[g_{1}, g_{2}, \ldots, g_{m}\right] \mid g_{i} \in \mathcal{G}_{i} \forall i \in \mathcal{I}\right\}
\end{aligned}
$$

is the range set for the multichannel image. Let a partial order relation $\leq$ be defined over each of the sets $\mathcal{G}_{i}$.

\section{DEFINITION 1}

The cartesian product set $\mathcal{G}$ is a poset under the relation $\preceq$; which is defined for all $F, G \in \mathcal{G}$ as $F \preceq G$ if and only if $f_{i} \leq g_{i}$ for $f_{i}, g_{i} \in \mathcal{G}_{i}$ and $i \in \mathcal{I}$.

A $\overline{\mathcal{G}}$-valued image $G$ on $E$ is a member of $\mathcal{L}=\mathcal{G}^{E}$ and is a map of the function $G: E \mapsto \mathcal{G}$. 


$$
G \stackrel{\text { def }}{=}\left\{G(x)=\left[g_{1}(x), g_{2}(x), \ldots, g_{m}(x)\right] \mid g_{i}(x) \in \mathcal{G}_{i} \forall i \in \mathcal{I}, x \in E\right\} .
$$

Hence the set $\mathcal{L}$ of all multivariate functions over the domain space $E$ and range space $\mathcal{G}$ represent multichanhel images and is as follows:

$$
\mathcal{L}=\mathcal{G}^{E}=\left(\prod_{i \in \mathcal{I}} \mathcal{G}_{i}\right)^{E} \cong \prod_{i \in \mathcal{I}} \mathcal{G}_{i}^{E}=\prod_{i \in \mathcal{I}} \mathcal{L}_{i}
$$

where $\mathcal{L}_{i}=\mathcal{G}_{i}^{E}$.

We will be dealing specifically with sets $\mathcal{G}_{i}$ that are complete lattices (in particular, complete chains). Therefore, $\mathcal{L}_{i}$ is also a complete lattice (also complete chain). We will henceforth call $\mathcal{L}_{i}$ as component lattice as they are members of the cartesian product set. The following theorem (Birkhoff 1973) establishes the structure of the object space (i.e., multichannel image under consideration). It should be noted that the manner in which the supremum and infimum operations are defined, it essentially induces marginal ordering on the multichannel image.

Theorem 1. The direct product $\mathfrak{R}=(\mathcal{L}, \sqcup, \Pi)$ of component lattices $\mathfrak{L}_{i}=\left(\mathcal{L}_{i}, \vee, \wedge\right)$ for $i \in \mathcal{I}$ is a complete lattice where for all $F, G \in \mathcal{L}=\prod_{i \in \mathcal{I}} \mathcal{L}_{i}$ we have:

$$
\begin{aligned}
& F \cup G=\left[f_{1} \vee g_{1}, f_{2} \vee g_{2}, \ldots, f_{m} \vee g_{m}\right] ; \\
& F \sqcap G=\left[f_{1} \wedge g_{1}, f_{2} \wedge g_{2}, \ldots, f_{m} \wedge g_{m}\right] .
\end{aligned}
$$

\section{COROLLARY 1.}

The direct product $\mathfrak{R}=(\mathcal{L}, \sqcup, \Pi)$ of component chains $\mathfrak{R}_{i}=\left(\mathcal{L}_{i}, \vee, \wedge\right)$ for $i \in \mathcal{I}$ is a complete chain.

\section{COROLLARY 2.}

The complete chain $\mathfrak{Q}=(\mathcal{L}, \sqcup, \Pi)$ is a completely distributive lattice.

\subsection{Matrix operators}

Having established the basic algebraic structure of a multichannel image (that of a complete lattice), we proceed to characterize the structure and properties of mappings between such complete lattices. Therefore for the discussion to follow in this section, we will be concerned with complete lattices $\mathfrak{R}_{a}, \mathfrak{Q}_{b}, \mathfrak{R}_{c}$ with the corresponding cartesian product sets $\mathcal{L}_{a}=\prod_{i \in \mathcal{I}} \mathcal{L}_{a, i} ; \mathcal{L}_{b}=\prod_{j \in \mathcal{J}} \mathcal{L}_{b, j} ; \mathcal{L}_{c}=\prod_{k \in \mathcal{K}} \mathcal{L}_{c, k}$, and $\mathcal{I}, \mathcal{J}, \mathcal{K}$ the indexing sets for the component sets $\mathcal{L}_{a, i}, \mathcal{L}_{b, j}, \mathcal{L}_{c, k}$. Here $\mathcal{I}=\{1,2, \ldots, m\} ; \mathcal{J}=\{1,2, \ldots, n\}$ and $\mathcal{K}=\{1,2, \ldots, p\}$. In order to prevent any undue increase in notational complexity, we will be using the same set of symbols for operations for the three lattices defined above. We will use $\preceq(\leq)$ for order relation, $\sqcup(\vee)$ for the supremum and $\Pi(\wedge)$ for the infimum in direct product lattices (component lattices). The least and the greatest element in $\mathcal{L}_{a}$ are $O_{a}=\left[O_{a, 1}, O_{a, 2}, \ldots, O_{a, m}\right]$ and $I_{a}=\left[I_{a, 1}, I_{a, 2}, \ldots, I_{a, m}\right]$ respectively (with $O_{a, i}$ and $I_{a, i}$ as the least and the greatest elements in $\mathcal{L}_{a, i}$ for all $i \in \mathcal{I}$ ). In a similar fashion, the least and the greatest elements in $\mathcal{L}_{b}$ and $\mathcal{L}_{c}$ are defined.

Let $\mathcal{M}_{a \mapsto b}=\mathcal{L}_{b}^{\mathcal{L}_{a}}$ represent the set of all mappings from $\mathcal{L}_{a} \mapsto \mathcal{L}_{b}$. It should be noticed here, that the elements of $\mathcal{M}_{a \mapsto b}$ are matrix operators with each entity (element) of the matrix being an operator between component lattices. Let $\Psi \in \mathcal{M}_{a \rightarrow b}$ be an $m \times n$ matrix operator with $\Psi=\left[\psi_{i, j} ; i \in \mathcal{I}, j \in \mathcal{J}\right]$ and $\psi_{i, j} \in \mathcal{L}_{b, j}^{\mathcal{L}_{a, i}}$, that is $\psi_{i, j}: \mathcal{L}_{a, i} \mapsto \mathcal{L}_{b, j}$. It is worth noting that if for any $\Psi \in \mathcal{M}_{a \mapsto b}$, then $\Psi^{T} \in \mathcal{M}_{b \mapsto a}$. 


\section{DEFINITION 2}

The order relation $\preceq$ on elements of $\mathcal{M}_{a \mapsto b}$ is defined for all $\Phi, \Psi \in \mathcal{M}_{a \mapsto b}$, as $\Phi \preceq \Psi$ if and only if $\phi_{i, j} \leq \psi_{i, j}$ for all $i \in \mathcal{I}$ and $j \in \mathcal{J}$.

As a result of $\mathcal{L}_{b, j}$ for all $j \in \mathcal{J}$ being a complete lattice, the set of all mappings $\mathcal{L}_{b, j}^{\mathcal{L}_{a, i}}$ for all $i \in \mathcal{I}$ and $j \in \mathcal{J}$ has the structure of a complete lattice. This in turn induces a complete lattice structure to the set $\mathcal{M}_{a \mapsto b}$ of matrix operators. We shall denote supremum and infimum in the set $\mathcal{M}_{a \mapsto b}$ by $\sqcup$ and $\sqcap$ respectively. Here $\left(\mathcal{M}_{a \mapsto b}, \preceq\right)$ has least element $\Xi$ and the greatest element $\Gamma$ defined as below.

\section{DEFINITION 3}

Matrix constant operators are defined as:

$$
\begin{aligned}
& \Gamma=\left[\gamma_{i, j} ; i \in \mathcal{I}, j \in \mathcal{J}\right], \quad \text { where } \quad \gamma_{i, j}\left(f_{i}\right)=I_{b, j} ; \\
& \Xi=\left[\xi_{i, j} ; i \in \mathcal{I}, j \in \mathcal{J}\right], \quad \text { where } \xi_{i, j}\left(f_{i}\right)=O_{b, j} .
\end{aligned}
$$

Hence for any $F \in \mathcal{L}_{a}$, we have $\Gamma(F)=I_{b}$ and $\Xi(F)=O_{b}$. We next give the definition of how a matrix operator from $\mathcal{M}_{a \mapsto b}$ operates on the elements of the set $\mathcal{L}_{a}$.

\section{DEFINITION 4}

For $\Psi \in \mathcal{M}_{a \mapsto b}, F \in \mathcal{L}_{a}$ and $G \in \mathcal{L}_{b}$, we define:

(a) $\Psi(F) \stackrel{\text { def }}{=} F \nabla \Psi=G$ where $g_{j}=\bigvee_{i \in \mathcal{I}} \psi_{i, j}\left(f_{i}\right)$ for all $j \in \mathcal{J}$;

(b) $\Psi(F) \stackrel{\text { def }}{=} F \Delta \Psi=G$ where $g_{j}=\bigwedge_{i \in \mathcal{I}} \psi_{i, j}\left(f_{i}\right)$ for all $j \in \mathcal{J}$.

In the sequel, whenever we use the notation $\Psi(F)$, then it would imply that we are referring to both kinds of matrix operations as defined above.

\section{PROPOSITION 1.}

For $\Phi, \Psi \in \mathcal{M}_{a \mapsto b}$ and $F \in \mathcal{L}_{a}$, we have:

$$
\Phi \preceq \Psi \quad \Rightarrow \quad \Phi(F) \preceq \Psi(F) .
$$

Thus for any $M \subseteq \mathcal{M}_{a \mapsto b}$ and $F \in \mathcal{L}_{a}$, it is a straightforward to show the following

$$
\begin{aligned}
& F \nabla\left(\bigsqcup^{M}\right)=\bigsqcup_{\Psi \in M}(F \nabla \Psi) \\
& F \Delta\left(\bigsqcup^{M}\right) \succeq \bigsqcup_{\Psi \in M}(F \Delta \Psi) \\
& F \nabla\left(\sqcap^{M}\right) \preceq \prod_{\Psi \in M}(F \nabla \Psi) \\
& F \Delta\left(\sqcap^{M}\right)=\prod_{\Psi \in M}(F \Delta \Psi) .
\end{aligned}
$$

Based on the previous discussion, we define three different types of composition between matrix operators.

\section{DEFINITION 5}

Matrix operators can be composed in the following way:

(a) For $\Psi, \Phi \in \mathcal{M}_{a \mapsto b}$, we define

$$
(\Psi \Phi) \stackrel{\text { def }}{=} \Phi \diamond \Psi=\left[\left(\psi_{i, j} \phi_{i, j}\right) ; i \in \mathcal{I}, j \in \mathcal{J}\right] \in \mathcal{M}_{a \mapsto b} ;
$$


(b) For $\Phi \in \mathcal{M}_{a \mapsto b}, \Psi \in \mathcal{M}_{b \mapsto c}$, we define

$$
(\Psi \Phi) \stackrel{\text { def }}{=} \Phi \nabla \Psi=\left[\bigvee_{j \in \mathcal{J}}\left(\psi_{j, k} \phi_{i, j}\right) ; i \in \mathcal{I}, k \in \mathcal{K}\right] \in \mathcal{M}_{a \mapsto c}
$$

(c) For $\Phi \in \mathcal{M}_{a \mapsto b}, \Psi \in \mathcal{M}_{b \mapsto c}$, we define

$$
(\Psi \Phi) \stackrel{\text { def }}{=} \Phi \Delta \Psi=\left[\bigwedge_{j \in \mathcal{J}}\left(\psi_{j, k} \phi_{i, j}\right) ; i \in \mathcal{I}, k \in \mathcal{K}\right] \in \mathcal{M}_{a \mapsto c}
$$

Here it should be noticed that for $F \in \mathcal{L}_{a} ;(\Psi \Phi)(F)$ is not necessarily the same as $\Psi(\Phi(F))$. Further, the process of matrix operator composition is possible only between compatible operators (i.e., matrix operators with compatible dimensions for composition). Thus for definition 5(a); $\Psi, \Phi$ and $\Phi \diamond \Psi$ are $m \times n$ matrices and for definition $5(\mathrm{~b}, \mathrm{c}), \Phi$ is $m \times n ; \Psi$ is $n \times p$ and $(\Psi \Phi)$ (i.e., $\Phi \nabla \Psi$ and $\Phi \Delta \Psi$ ) is $m \times p$. It is evident that composition of matrix operators is associative but not commutative. Further, it can be shown as a result of the ordering of matrix operators that for all $\Phi, \Psi, \Theta \in \mathcal{M}_{a \mapsto b}$ such that $\Phi \preceq \Psi$ implies $\Theta \diamond \Phi \preceq \Theta \diamond \Psi$. Similarly, for $\Theta \in \mathcal{M}_{a \mapsto b}$ and for all $\Phi, \Psi \in \mathcal{M}_{b \mapsto c}$ such that $\Phi \preceq \Psi$ implies that $\Theta \nabla \Phi \preceq \Theta \nabla \Psi$ and $\Theta \Delta \Phi \preceq \Theta \Delta \Psi$ which is equivalent to stating that $(\Phi \Theta) \preceq(\Psi \Theta)$. For a general case, with $\Psi \in \mathcal{M}_{a \mapsto b}$ and $M \subseteq \mathcal{M}_{a \mapsto b}$ we have:

$$
\begin{aligned}
& \Psi \diamond\left(\bigsqcup^{M}\right)=\bigsqcup_{\Phi \in M}(\Psi \diamond \Phi) \\
& \Psi \diamond\left(\prod^{M}\right)=\prod_{\Phi \in M}(\Psi \diamond \Phi) .
\end{aligned}
$$

On the other hand, for any $\Psi \in \mathcal{M}_{a \mapsto b}$ and $M \subseteq \mathcal{M}_{b \mapsto c}$, we have:

$$
\begin{aligned}
& \Psi \nabla(\bigsqcup M)=\bigsqcup_{\Phi \in M}(\Psi \nabla \Phi) \\
& \Psi \Delta(\bigsqcup M) \succeq \bigsqcup_{\Phi \in M}(\Psi \Delta \Phi) \\
& \Psi \nabla\left(\prod^{M}\right) \preceq \prod_{\Phi \in M}(\Psi \nabla \Phi) \\
& \Psi \Delta\left(\prod^{M}\right)=\prod_{\Phi \in M}(\Psi \Delta \Phi) .
\end{aligned}
$$

\subsection{Matrix increasing operators}

We next describe matrix increasing operators and show that matrix dilation operator and matrix erosion operator are examples of increasing operators. Further matrix dilation and matrix erosion are dual operators in a lattice theoretic sense.

\section{DEFINITION 6}

For any $\Phi \in \mathcal{M}_{a \mapsto b}$, we define the following:

(a) The operator $\Phi$ is a matrix increasing operator if and only if $\phi_{i, j}$ for all $i \in \mathcal{I}, j \in \mathcal{J}$ are increasing operators.

(b) The operator $\Phi$ is a matrix dilation operator if and only if $\phi_{i, j}$ for all $i \in \mathcal{I}, j \in \mathcal{J}$ are dilation operators and the matrix operator operates according to the definition 4 (a). 
(c) The operator $\Phi$ is a matrix erosion operator if and only if $\phi_{i, j}$ for all $i \in \mathcal{I}, j \in \mathcal{J}$ are erosion operators and the matrix operator operates according to the definition 4(b).

Matrix dilation operators will be denoted by $\mathcal{D}$ with each entry as $\delta_{i, j}$ for all $i \in \mathcal{I}$ and $j \in \mathcal{J}$. Matrix erosion operators will be denoted by $\mathcal{E}$ with each entry as $\varepsilon_{i, j}$ for all $i \in \mathcal{I}$ and $j \in \mathcal{J}$.

\section{PROPOSITION 2.}

For any $\Phi \in \mathcal{M}_{a \mapsto b}$, such that it is a matrix increasing operator, then the following statements hold and are equivalent.

(a) For every $F, G \in \mathcal{L}_{a}$ such that $F \preceq G$ implies that $\Phi(F) \preceq \Phi(G)$.

(b) For any $S \subseteq \mathcal{L}_{a}$, we have $\Phi(\sqcup S) \succeq \bigsqcup_{F \in S} \Phi(F)$.

(c) For any $S \subseteq \mathcal{L}_{a}$, we have $\Phi(\sqcap S) \preceq \prod_{F \in S} \Phi(F)$.

\section{PROPOSITION 3.}

(a) Let $\mathcal{D} \in \mathcal{M}_{a \mapsto b}$ be a matrix dilation operator, then for any $S \subseteq \mathcal{L}_{a}$, the following holds:

$$
(\bigsqcup S) \nabla \mathcal{D}=\bigsqcup_{F \in S}(F \nabla \mathcal{D}) .
$$

(b) Let $\mathcal{E} \in \mathcal{M}_{a \mapsto b}$ be a matrix erosion operator, then for any $S \subseteq \mathcal{L}_{a}$, the following holds:

$$
\left(\prod S\right) \Delta \mathcal{E}=\prod_{F \in S}(F \Delta \mathcal{E})
$$

It is evident that as $\varepsilon_{i, j}$ and $\delta_{i, j}$ for all $i \in \mathcal{I}, j \in \mathcal{J}$ are increasing operators, matrix erosion operator $\mathcal{E}$ and matrix dilation operator $\mathcal{D}$ are matrix increasing operators.

The following facts follow as a consequence of the above discussion and are properties of operator composition analogous to (5) and (6).

- Let $\Theta$ be a matrix increasing operator, then for any $\Phi, \Psi \in \mathcal{M}_{a \mapsto b}$ such that $\Phi \preceq \Psi$ implies $(\Theta \Phi) \preceq(\Theta \Psi)$.

- Let $\Theta$ be a matrix dilation operator and $M \subseteq \mathcal{M}_{a \mapsto b}$, then

$$
\begin{aligned}
& \left(\Theta\left(\bigsqcup^{M}\right)\right)=\bigsqcup_{\Psi \in M}(\Theta \Psi) \\
& \left(\Theta\left(\Pi^{M}\right)\right) \preceq \prod_{\Psi \in M}(\Theta \Psi) .
\end{aligned}
$$

- Let $\Theta$ be a matrix erosion operator and $M \subseteq \mathcal{M}_{a \mapsto b}$, then

$$
\left(\Theta\left(\bigsqcup^{M}\right)\right) \succeq \bigsqcup_{\Psi \in M}(\Theta \Psi)
$$




$$
\left(\Theta\left(\Pi^{M}\right)\right)=\prod_{\Psi \in M}(\Theta \Psi) .
$$

Here it should be kept in mind that operator composition results given above are valid for appropriate dimension of the operator $\Theta$, i.e., $\Theta \in \mathcal{M}_{a \mapsto b}$ for composition operator $\diamond$ and $\Theta \in \mathcal{M}_{b \mapsto c}$ for composition operator $\nabla$ or $\Delta$.

\section{PROPOSITION 4.}

The set of matrix increasing operators from $\mathcal{L}_{a} \mapsto \mathcal{L}_{b}$ is closed under composition by $\diamond$ and is a complete sublattice of $\mathcal{M}_{a \mapsto b}$.

Since matrix diflation and matrix erosion are increasing operators, therefore they must satisfy results analogous to proposition 4 . This is stated in the following proposition.

\section{PROPOSITION 5.}

(a) The set of matrix dilation operators from $\mathcal{L}_{a} \mapsto \mathcal{L}_{b}$ is closed under composition by $\diamond$ and is a sup-closed subset of $\mathcal{M}_{a \mapsto b}$.

(b) The set of matrix erosion operators from $\mathcal{L}_{a} \mapsto \mathcal{L}_{b}$ is closed under composition by $\diamond$ and is a inf-closed subset of $\mathcal{M}_{a \mapsto b}$.

As a consequence, the set consisting of matrix dilation and matrix erosion operators is a complete lattice. The least matrix dilation operator is $\Xi$ and supremum of dilations is $\sqcup$. Dually, the greatest matrix erosion operator is $\Gamma$ and infimum of erosions is $\Pi$.

\section{PROPOSITION 6.}

Let $\mathcal{D} \in \mathcal{M}_{a \mapsto b}, \mathcal{E} \in \mathcal{M}_{b \mapsto a}$ be matrix increasing operators. If the pair $(\mathcal{E}, \mathcal{D})$ is an adjunction (i.e. $F \nabla \mathcal{D} \preceq G \Longleftrightarrow F \preceq G \Delta \mathcal{E}$ ), then $\mathcal{D}$ is an $m \times n$ matrix dilation operator and $\mathcal{E}$ is an $n \times m$ matrix erosion operator.

The above proposition relates to every erosion operator $\mathcal{E}$, a corresponding dilation operator $\mathcal{D}$ and vice versa. This pair $(\mathcal{E}, \mathcal{D})$ is called an adjunction with $\mathcal{E}$ being the upper adjoint and $\mathcal{D}$ the lower adjoint.

In the proposition to follow, we have: $\Lambda_{b}=\left[\lambda_{j_{1}, j_{2}}^{b} ; j_{1}, j_{2} \in \mathcal{J}\right]$ where $\lambda_{j_{1}, j_{2}}^{b}$ is equal to $\lambda_{j_{1}, j_{1}}^{b}$ for $j_{1}=j_{2}$ and $\gamma_{j_{1}, j_{2}}^{b}$ for $j_{1} \neq j_{2}$ such that $\lambda_{j_{1}, j_{1}}^{b}\left(f_{j_{1}}\right)=f_{j_{1}}$ and $\gamma_{j_{1}, j_{2}}^{b}\left(f_{j_{1}}\right)=I_{b, j_{2}}$. Similarly, we have $\Lambda_{a}=\left[\lambda_{i_{1}, i_{2}}^{a} ; i_{1}, i_{2} \in \mathcal{I}\right]$ where $\lambda_{i_{1}, i_{2}}^{a}$ is equal to $\lambda_{i_{1}, i_{1}}^{a}$ for $i_{1}=i_{2}$ and $\xi_{i_{1}, i_{2}}^{a}$ for $i_{1} \neq i_{2}$ such that $\lambda_{i_{1}, i_{1}}^{d}\left(f_{i_{1}}\right)=f_{i_{1}}$ and $\xi_{i_{1}, i_{2}}^{a}\left(f_{i_{1}}\right)=O_{a, i_{2}}$. Hence $F \Delta \Lambda_{b}=F$ for $F \in \mathcal{L}_{b}$ and $F \nabla \Lambda_{a}=F$ for $F \in \mathcal{L}_{a}$.

\section{PROPOSITION 7.}

For all $\mathcal{D} \in \mathcal{M}_{a \mapsto b}, \mathcal{E} \in \mathcal{M}_{b \rightarrow a}$ matrix increasing operators such that the pair $(\mathcal{E}, \mathcal{D})$ is an adjunction, then the following hold:

(a) $\mathcal{D} \Delta \mathcal{E} \succeq \Lambda_{a}$

(b) $\mathcal{E} \nabla \mathcal{D} \preceq \Lambda_{b}$

(c) $\mathcal{E} \nabla \mathcal{D} \Delta \mathcal{E}=\mathcal{E}$

(d) $\mathcal{D} \triangle \mathcal{E} \nabla \mathcal{D}=\mathcal{D}$

\section{PROPOSITION 8.}

(a) Given that $\left(\mathcal{E}_{1}, \mathcal{D}_{1}\right)$ and $\left(\mathcal{E}_{2}, \mathcal{D}_{2}\right)$ are adjunctions then $\mathcal{E}_{1} \succeq \mathcal{E}_{2}$ if and only if $\mathcal{D}_{1} \leq \mathcal{D}_{2}$. 
(b) Given that the pairs $\left(\mathcal{E}^{(t)}, \mathcal{D}^{(t)}\right)$ are adjunctions for all $t \in \mathcal{T}$, then $\left(\Pi_{t \in \mathcal{I}} \mathcal{E}^{(t)}\right.$, $\left.\sqcup_{t \in \mathcal{T}} \mathcal{D}^{(t)}\right)$ is also an adjunction.

(c) Given a pair of matrix dilation operators $\mathcal{D}_{1}, \mathcal{D}_{2} \in \mathcal{M}_{a \mapsto b}$ and a pair of matrix erosion operators $\mathcal{E}_{1}, \mathcal{E}_{2} \in \mathcal{M}_{b \mapsto a}$, such that the pairs $\left(\mathcal{E}_{1}, \mathcal{D}_{1}\right)$ and $\left(\mathcal{E}_{2}, \mathcal{D}_{2}\right)$ are adjunctions, then $\left(\mathcal{E}_{1} \Delta \mathcal{E}_{2}, \mathcal{D}_{2} \nabla \mathcal{D}_{1}\right)$ is an adjunction.

\section{DEFINITION 7}

A projection map $\mathcal{P}_{i}: \mathcal{L} \mapsto \mathcal{L}_{i}$ for all $i \in \mathcal{I}$ from a direct product complete lattice $\mathcal{L}$ to the $i$ th component $\mathcal{L}_{i}$ is defined as $\mathcal{P}_{i}(F)=f_{i}$ for all $i \in \mathcal{I}$ with $F=\left[f_{1}\right.$, $\left.f_{2}, \ldots, f_{m}\right] \in \mathcal{L}$ and $f_{i} \in \mathcal{L}_{i}$.

The projection map or operator plays an important role in certain cases. These projection operators can also be defined in terms of matrix operators, i.e., matrix dilation/erosion operators can be obtained for each projection operator.

Let $\mathcal{D}_{\mathcal{P}_{i}}=\left[\xi_{i, 1} \xi_{i, 2} \cdots \lambda_{i, i} \cdots \xi_{i, m}\right]^{T}$ and $\mathcal{E}_{\mathcal{P}_{i}}=\left[\gamma_{i, 1} \gamma_{i, 2} \cdots \lambda_{i, i} \cdots \gamma_{i, m}\right]^{T}$ for all $i \in \mathcal{I}$. Here $\xi_{i, i^{\prime}}\left(f_{i}\right)=O_{i^{\prime}}$ and $\gamma_{i, i^{\prime}}\left(f_{i}\right)=I_{i^{\prime}}$ for all $i, i^{\prime} \in \mathcal{I}$ and $i \neq i^{\prime}$ and $\lambda_{i, i}\left(f_{i}\right)=f_{i}$ for all $i \in \mathcal{I}$. With these operators, we have $F \nabla \mathcal{D}_{\mathcal{P}_{i}}=f_{i}$ and $F \Delta \mathcal{E}_{\mathcal{P}_{i}}=f_{i}$. Next we define two operators $\mathcal{E}^{\prime}{ }_{\mathcal{P}_{i}}: \mathcal{L}_{i} \mapsto \mathcal{L}$ and $\mathcal{D}^{\prime}{ }_{\mathcal{P}_{i}}: \mathcal{L}_{i} \mapsto \mathcal{L}$ such that $\mathcal{E}^{\prime}{ }_{\mathcal{P}_{i}}=\left[\gamma_{1, i} \gamma_{2, i} \cdots \lambda_{i, i} \cdots \gamma_{m, i}\right]$ $\mathcal{D}^{\prime}{ }_{\mathcal{P}_{i}}=\left[\xi_{1, i} \xi_{2, i} \cdots \lambda_{i, i} \cdots \xi_{m, i}\right]$. We see that $f_{i} \Delta \mathcal{E}_{\mathcal{P}_{i}}^{\prime}=\left[I_{1}, I_{2}, \ldots, f_{i}, \ldots, I_{m}\right]$ and $f_{i} \nabla \mathcal{D}_{\mathcal{P}_{i}}^{\prime}=\left(O_{1}, O_{3}, \ldots, f_{i}, \ldots, O_{m}\right)$.

\section{PROPOSITION 9.}

For the above defined operators $\mathcal{E}_{\mathcal{P}_{i}}$ and $\mathcal{D}_{P_{i}}$ from $\mathcal{L} \mapsto \mathcal{L}_{i}$ and $\mathcal{E}^{\prime}{ }_{\mathcal{P}_{i}}$ and $\mathcal{D}^{\prime}{ }_{P_{i}}$ from $\mathcal{L}_{i} \mapsto \mathcal{L}$, then the pairs $\left(\mathcal{E}_{\mathcal{P}_{i}}, \mathcal{D}^{\prime}{ }_{\mathcal{P}_{i}}\right)$ and $\left(\mathcal{E}^{\prime}{ }_{\mathcal{P}_{i}}, \mathcal{D}_{\mathcal{P}_{i}}\right)$ are adjunctions.

\section{Translation invariant operators}

In this section, we are concerned with multivariate morphological operators that are translation invariant. We will only discuss spatial translation invariance and will not consider gray level translation invariance. Towards this end, we will work with a completely lattice ordered commutative (clc) monoid structure for image as used by Hseuh (1992) and Singh \& Siddiqi (1995) for the univariate case. A part of the results being presented in this section have already appeared (Wilson 1992) in the context of matrix morphology. However, these results as presented by Wilson (1992) are only applicable to the binary and infinite gray-level case and have been proved using concepts from set theory. On the other hand, the results detailed out here are applicable to any chain structure and have been proved using results from the theory of lattice ordered monoids. These results are generalized versions of matrix morphology and are applicable in a larger gamut of situations.

We have assumed in the previous section that $\mathfrak{L}=(\mathcal{L}, \sqcup, \Pi)$ is a complete lattice. Here, we additionally assume that $\mathcal{L}_{i}=\mathcal{G}_{i}^{E}$ and $\mathcal{G}_{i}=\mathcal{G}$ for all $i \in \mathcal{I}$ and a binary operation $\star$ is defined on these component sets such that $\left(\mathcal{L}_{i}, \star\right)$ is a commutative monoid. Further the domain set $E$ is assumed to possess an abelian group structure under the binary operation + .

\section{PROPOSITION 10.}

The direct product $(\mathcal{L}, \star)$ of component commutative monoids $\left(\mathcal{L}_{i}, \star\right)$ is itself a commutative monoid under the pointwise binary operation defined for all $F, G \in \mathcal{L}$ as

$$
F \star G=\left[f_{1} \star g_{1}, f_{2} \star g_{2}, \ldots, f_{m} \star g_{m}\right]
$$

for all $f_{i}, g_{i} \in \mathcal{L}_{i}$ with $i \in \mathcal{I}$. 
Theorem 2. The direct product structure $(\mathcal{L}, \sqcup, \Pi, \star)$ is a clc-monoid.

The least element of $\mathcal{L}$ is $O$ which implies $\sqcup \varphi=O$. Therefore for all $F \in \mathcal{L}$, we have $F \star O=F \star(\sqcup \varphi)=\sqcup_{G \in \varphi}(F \star G)=\sqcup \varphi=O$. Every $c l c$-monoid is residuated and the residuation is given for all $F, G \in \mathcal{L}$ as

$$
F: G=\bigsqcup\{H \in \mathcal{L} \mid G \star H \preceq F\} .
$$

Thus the structure of a multivariate signal is that of $c l c$-monoid. The dilation $\otimes$ and erosion operators on univariate signals have already been defined (Hseuh 1992; Singh \& Siddiqi 1995). We now define multivariate operators in the following.

\section{DEFINITION 8}

(a) A matrix dilation operator is defined as:

$$
H=\mathcal{D}_{G}(F)=F \text { 团 } G \quad \text { where } \quad h_{j}=\bigvee_{i \in \mathcal{I}}\left(f_{i} \otimes g_{i, j}\right) \quad \text { for all } j \in \mathcal{J}
$$

(b) A matrix erosion operator is defined as:

$$
H=\mathcal{E}_{G}(F)=F \boxminus G \quad \text { where } \quad h_{j}=\bigwedge_{i \in \mathcal{I}}\left(f_{i} \odot g_{i, j}\right) \quad \text { for all } j \in \mathcal{J}
$$

(c) Matrix dual operator is defined as $G^{d}=\left[g_{i, j}^{d} ; i \in \mathcal{I}, j \in \mathcal{J}\right]$, where for $g, h \in \mathcal{L}$ we have $g \leq h$ if and only if $h^{d} \leq g^{d}$ and $\left(g^{d}\right)^{d}=g$.

(d) Matrix reflected-dual operator is defined as $G^{*}=\left[g_{i, j}^{*} ; i \in \mathcal{I}, j \in \mathcal{J}\right]$, where $g^{*}(x)=$ $g^{d}(-x)$.

(e) Matrix translated operator is defined as $G^{\tau}=\left[g_{i, j}^{\tau} ; i \in \mathcal{I}, j \in \mathcal{J}\right]$, where $g^{\tau}(x)=$ $g(x-\tau)$.

(f) Transpose of a matrix operator is defined as $G^{T}=\left[g_{j, i} ; i \in \mathcal{I}, j \in \mathcal{J}\right]$.

In the above definitions, $F$ is a multichannel image and $G$ is a matrix structuring element. In the following, we state the duality and translation invariance of matrix operators.

PROPOSITION 11.

Let $(\mathcal{L}, \sqcup, \Pi, \circledast)$ be a self dual clc-monoid $\left(\right.$ i.e. $\left.f^{d} \circledast g=(f \odot g)^{d}\right)$, then

(a) $\left(F^{*} \text { 因 } G\right)^{*}=F[G$

(b) $\left(F^{*} \boxminus G\right)^{*}=F$ 因

\section{PROPOSITION 12.}

For any matrix operators, the following holds

(a) $F^{\tau}$ 因 $G=(F \text { 因 } G)^{\tau}=F$ 因 $G^{\tau}$

(b) $F^{\tau} \boxminus G=(F \boxminus G)^{\tau}=F \boxminus G^{-\tau}$.

The increasing property of matrix operators is described in the next proposition.

PROPOSITION 13.

(a) For all $F, G \in \mathcal{L}, F \preceq G$ implies $F$ 因 $H \preceq G$ 因 $H$.

(b) For all $H, H^{\prime} \in \mathcal{M}$ and $F \in \mathcal{L}, H \preceq H^{\prime}$ implies $F$ 因 $H \preceq F$ 因 $H^{\prime}$. 
(c) For all $F, G \in \mathcal{L}, F \preceq G$ implies $F \square H \preceq G \boxminus H$.

(d) For all $H, H^{\prime} \in \mathcal{M}$ and $F \in \mathcal{L}, H \preceq H^{\prime}$ implies $F\left[H \preceq F \boxminus H^{\prime}\right.$.

The next proposition states eight types of distributive laws for matrix operators. PROPOSITION 14.

(a) $F$ 因 $\left(\bigsqcup_{t \in \mathcal{T}} G^{(t)}\right)=\bigsqcup_{t \in \mathcal{T}}\left(F\right.$ 团 $\left.G^{(t)}\right)$

(b) $F \otimes\left(\prod_{t \in \mathcal{T}} G^{(t)}\right) \preceq \prod_{t \in \mathcal{T}}\left(F\right.$ 因 $\left.G^{(t)}\right)$

(c) $\quad F \boxminus\left(\prod_{t \in T} G^{(t)}\right) \succeq \bigsqcup_{t \in T}\left(F \boxminus G^{(t)}\right)$

(d) $\quad F \boxminus\left(\bigsqcup_{t \in \mathcal{T}} G^{(t)}\right)=\prod_{t \in \mathcal{T}}\left(F \boxminus G^{(t)}\right)$

(e) $\left(\bigsqcup_{t \in \mathcal{T}} F^{(t)}\right) \boxplus G=\bigsqcup_{t \in \mathcal{T}}\left(F^{(t)}\right.$ 因G)

(f) $\left(\prod_{t \in \mathcal{T}} F^{(t)}\right) \otimes G \preceq \prod_{t \in \mathcal{T}}\left(F^{(t)} \boxplus G\right)$

(g) $\left(\bigsqcup_{t \in T} F^{(t)}\right) \boxminus G \succeq \bigsqcup_{t \in T}\left(F^{(t)} \boxminus G\right)$

(h) $\left(\prod_{t \in \mathcal{T}} F^{(t)}\right) \boxminus G=\prod_{t \in \mathcal{T}}\left(F^{(t)} \boxminus G\right)$

We next state results concerning matrix dilation and matrix erosion operators.

\section{PROPOSITION 15.}

(a) $F$ 因G $\leq H \quad \Longleftrightarrow \quad F \preceq H \boxminus G^{T}$.

(b) $(F \boxminus G)$ 团 $G^{T} \preceq F \preceq\left(F\right.$ 因G)曰G $G^{T}$.

(c) $(F$ 圆G)团H=F因 $(G$ 因 $H)$.

(d) $(F \square G) \square H=F \square(G \boxplus H)$.

(e) $F \boxminus(G \square H) \succeq(F \boxminus G)$ 团 $H$.

(f) $F$ 团 $(G \boxminus H) \leq(F$ 团G)【H.

In the following we discuss matrix opening and matrix closing operators.

DEFINITION 9
(a) Matrix opening operator is defined as $F \circ G=(F \boxminus G)$ 团 $G^{T}$.
(b) Matrix closing operator is defined as $F \bullet G=(F \boxplus G) \square G^{T}$.

(a) Duality of matrix opening and closing operator: 


$$
\left(F^{*} \circ G\right)^{*}=F \bullet G \text { and }\left(F^{*} \bullet G\right)^{*}=F \circ G \text {. }
$$

(b) Increasing properties: $F \preceq G \Rightarrow F \circ H \preceq G \circ H$ and $F \bullet H \preceq G \bullet H$.

(c) Opening operator is antiextensive $F \circ G \preceq F$ and closing operator is extensive $F \bullet G \succeq F$.

(d) Idempotence of matrix opening and closing operators:

$$
F \circ G=(F \circ G) \circ G \quad \text { and } \quad F \bullet G=(F \bullet G) \bullet G .
$$

Next we state some weak distributive laws concerning matrix opening and closing operators.

PROPOSITION 17.

(a) $\left(\bigsqcup_{t \in \mathcal{T}} F^{(t)}\right) \circ G \succeq \bigsqcup_{t \in \mathcal{T}}\left(F^{(t)} \circ G\right)$.

(b) $\left(\prod_{t \in \mathcal{T}} F^{(t)}\right) \circ G \preceq \prod_{t \in \mathcal{T}}\left(F^{(t)} \circ G\right)$.

(c) $\left(\bigsqcup_{t \in \mathcal{T}} F^{(t)}\right) \bullet G \succeq \bigsqcup_{t \in \mathcal{T}}\left(F^{(t)} \bullet G\right)$.

(d) $\left(\prod_{t \in \mathcal{T}} F^{(t)}\right) \bullet G \preceq \prod_{t \in \mathcal{T}}\left(F^{(t)} \bullet G\right)$.

\section{Quasi-lattices and morphological operators}

In earlier sections, we had imposed a partial ordering on the cartesian product set by componentwise ordering (i.e., marginal ordering) and subsequently obtained morphological matrix operators. Another possibility could have been to apply a linear transformation on the multichannel image followed by the application of multivariate morphological operators as discussed in $\$ 2$. Such an approach has been investigated by Eo (1992) and Goutisias et al (1994). This approach also utilizes the marginal ordering principle. However it should be realized that with the help of marginal ordering, not all elements of the cartesian product set can be compared. In order to overcome this limitation, one may define a mapping procedure such that all elements of the mapped set can somehow be compared. This is possible if some kind of reduced ordering is employed, since it imposes a total ordering on the set of multivariate data. This poses before us the following question: What should be the mapped set and what are the kind of mappings that must be considered? Since the elements of the mapped set should have a total ordering over its elements, one can choose the set $\mathbb{R}$. Let us assume that there exists a surjective mapping $\mathcal{Q}: \mathcal{L} \mapsto \mathbb{R}$. The actual mappings that are of interest will be considered later in this section. To begin with we give the following definitions.

DEFINITION 10

(a) The binary order relation $\preceq$ is defined for all $F, G \in \mathcal{L}$ as $F \preceq G$ if and only if $\mathcal{Q}(F) \leq \mathcal{Q}(G)$.

(b) The relation $\simeq$ is defined for all $F, G \in \mathcal{L}$ as $F \simeq G$ if and only if $F \preceq G$ and $G \preceq F$, that is $\mathcal{Q}(F)=\mathcal{Q}(G)$. 
PROPOSITION 18.

The structure $(\mathcal{L}, \preceq)$ is a quasi-ordered set.

PROPOSITION 19.

In a quasi-ordered set $(\mathcal{L}, \preceq)$, we have:

(a) The relation $\simeq$ is an equivalence relation on $\mathcal{L}$.

(b) If $\mathcal{C}_{1}$ and $\mathcal{C}_{2}$ are two equivalence classes corresponding to the relation $\simeq$, then $F_{1} \leq F_{2}$ either for no $F_{1} \in \mathcal{C}_{1}$ and $F_{2} \in \mathcal{C}_{2}$ or for all $F_{1} \in \mathcal{C}_{1}$ and $F_{2} \in \mathcal{C}_{2}$.

(c) The quotient set $\mathcal{S}=\mathcal{L} / \simeq$ is a poset where $\mathcal{C}_{1} \leq \mathcal{C}_{2}$ is defined to mean that $F \leq G$ for all $F \in \mathcal{C}_{1}$ and $G \in \mathcal{C}_{2}$.

The equivalence class is defined as $\mathcal{C}(f)=\{F \in \mathcal{L} \mid \mathcal{Q}(F)=f \in \overline{\mathbb{R}}\}$. There exists mappings $\mathcal{Q}^{-1}: \overline{\mathbb{R}} \mapsto \mathcal{L}$ such that $\mathcal{Q} \mathcal{Q}^{-1}(f)=f$ for all $f \in \overline{\mathbb{R}}$. Here $\mathcal{Q} \mathcal{Q}^{-1}$ is the identity operator $\lambda$. However it should be noted that $\mathcal{Q}^{-1} \mathcal{Q}$ is not necessarily the identity operator $\Lambda$. However $\mathcal{Q Q}^{-1} \mathcal{Q}=\mathcal{Q}$. Hence the operator $\mathcal{Q}^{-1}$ is termed as the pseudoinverse of $\mathcal{Q}$. Thus a quasi-ordering relation is imposed on the set $\mathcal{L}$ with the help of a surjective map $\mathcal{Q}$ and the order on $\overline{\mathbb{R}}$. In a similar manner, one can define supremum $\sqcup$ and infimum $\Pi$ of any subset of $\mathcal{L}$. Hence we have

$$
\begin{aligned}
\mathcal{Q}\left(\bigsqcup_{t \in \mathcal{T}}\left(F^{(t)}\right)\right) & =\bigvee_{t \in \mathcal{T}} \mathcal{Q}\left(F^{(t)}\right)=\mathcal{Q}\left(F^{(s)}\right) \\
\Rightarrow \quad \bigsqcup_{t \in \mathcal{T}}\left(F^{(t)}\right) & \simeq F^{(s)} .
\end{aligned}
$$

Similarly, we also have

$$
\begin{aligned}
& \mathcal{Q}\left(\prod_{t \in \mathcal{T}}\left(F^{(t)}\right)\right)=\bigwedge_{t \in \mathcal{T}} \mathcal{Q}\left(F^{(t)}\right)=\mathcal{Q}\left(F^{(s)}\right) \\
& \Rightarrow \quad \prod_{t \in \mathcal{T}}\left(F^{(t)}\right) \simeq F^{(s)} .
\end{aligned}
$$

Here we refer to $(\mathcal{L}, \sqcup, \Pi)$ as a complete quasi-lattice since $\mathcal{L}$ is a quasi-ordered set.

We are now in a position to obtain morphological operators over complete quasi-lattices. However it should be realized at this point that these operators are essentially over equivalence classes of $\mathcal{L}$. This is because any operator has to take all elements from one equivalence class to elements of some other equivalence class. The quasi-ordering relation $\preceq$ on $\mathcal{L}$ induces a quasi ordering on the operators over the set $\mathcal{L}$ as $\Phi \leq \Psi$ if and only if $\Phi(F) \preceq \Psi(F)$ for all $F \in \mathcal{L}$. It is evident that the set of operators mapping $\mathcal{L}$ to $\mathcal{L}$ is also a quasi-ordered set. An equivalence relation can also be defined on operators. We say that $\Phi \simeq \Psi$ if and only if $\mathcal{Q}(\Phi)=\mathcal{Q}(\Psi)$. This equivalence relation partitions the set of quasi-increasing operators into equivalence classes.

\section{DEFINITION 11}

(a) An operator $\Psi: \mathcal{L} \mapsto \mathcal{L}$ is a quasi-increasing operator if for all $F, G \in \mathcal{L}, F \preceq G$ implies $\Psi(F) \preceq \Psi(G)$.

(b) A pair of operators $(\mathcal{E}, \mathcal{D})$ is called a quasi-adjunction if for all $F, G \in \mathcal{L}$ we have:

$$
\mathcal{D}(F) \preceq G \quad \Longleftrightarrow \quad F \preceq \mathcal{E}(G) .
$$


The set of quasi-increasing operators is closed under operator composition. Further if the pair of operators $(\mathcal{E}, \mathcal{D})$ is a quasi-adjunction, then it is straightforward to verify that $\mathcal{E}$ and $\mathcal{D}$ are quasi-increasing operators. It should be noted that $\mathcal{Q}^{-1} \mathcal{Q} \simeq \Lambda$ and is a quasiincreasing operator. Thus we have the following proposition.

PROPUSITION 20.

Let $\Psi$ be a quasi-increasing operator on $\mathcal{L}$, then we have

$$
\Psi \mathcal{Q}^{-1} \mathcal{Q} \simeq \Psi \simeq \mathcal{Q}^{-1} \mathcal{Q} \Psi
$$

\section{PROPOSITION 21.}

An operator $\Psi: \mathcal{L} \mapsto \mathcal{L}$ is quasi-increasing if and only if there exists an operator $\psi: \mathbb{\mathbb { R }} \mapsto \mathbb{\mathbb { R }}$ such that $\psi \mathcal{Q}=\mathcal{Q} \Psi$. Further the operator $\psi$ can be uniquely determined from $\Psi$ as $\psi=\mathcal{Q} \Psi \mathcal{Q}^{-1}$, where $\mathcal{Q}^{-1}$ is any arbitrary pseudo inverse of $\mathcal{Q}$.

The readers interested in the proof of the above proposition can refer to Goutsias et al (1994). We observe that there are many operators $\Psi$ which will map to the same operator $\psi$. All these operators are said to belong to an equivalence class of operators defined as $\mathcal{C}(\psi)=\left\{\Psi \mid \psi=\mathcal{Q} \Psi \mathcal{Q}^{-1}\right\}$. We can now state the following proposition.

\section{PROPOSITION 22.}

For any $\Psi \in \mathcal{C}(\psi)$ and $\Phi \stackrel{\text { def }}{=} \mathcal{Q}^{-1} \mathcal{Q} \Psi$ for any arbitrary pseudo-inverse $\mathcal{Q}^{-1}$, then $\Phi \in$ $\mathcal{C}(\psi)$.

Hence one can obtain all the elements of $\mathcal{C}(\psi)$ by composing any element $\Psi \in \mathcal{C}(\psi)$ with $\mathcal{Q}^{-1} \mathcal{Q}$ for all possible pseudo-inverse operators $\mathcal{Q}^{-1}$.

\section{PROPOSITION 23.}

The pair of operators $(\mathcal{E}, \mathcal{D})$ is a quasi-adjunction on $\mathcal{L}$ for all $\mathcal{E} \in \mathcal{C}(\varepsilon)$ and $\mathcal{D} \in \mathcal{C}(\delta)$ if and only if the pair $(\varepsilon, \delta)$ is an adjunction on $\overline{\mathbb{R}}$.

Since $\varepsilon$ and $\delta$ are erosion and dilation operators on $\overline{\mathbb{R}}$, therefore we term $\mathcal{E}$ and $\mathcal{D}$ as quasi-erosion and quasi-dilation operators respectively. In order to illustrate how the results for erosion and dilation operators over complete lattices can be modified to corresponding results related to quasi-erosion and quasi-dilation operators over complete quasi-lattices, we state the following proposition.

\section{PROPOSITION 24.}

Let $(\mathcal{E}, \mathcal{D})$ be a quasi-adjunction between $\mathcal{L}$ and $\mathcal{L}$ then we have the following:

(a) $\mathcal{D}\left(\sqcup_{t \in \mathcal{T}} F^{(t)}\right) \simeq \sqcup_{t \in \mathcal{T}} \mathcal{D}\left(F^{(t)}\right)$ for $\left\{F^{(t)} \in \mathcal{L} ; t \in \mathcal{T}\right\}$.

(b) $\mathcal{E}\left(\Pi_{t \in \mathcal{T}} F^{(t)}\right) \simeq \Pi_{t \in \mathcal{T}} \mathcal{E}\left(F^{(t)}\right)$ for $\left\{F^{(t)} \in \mathcal{L} ; t \in \mathcal{T}\right\}$.

(c) $\mathcal{E}(I) \simeq I$ and $\mathcal{D}(O) \simeq O$.

(d) $\mathcal{E D} \succeq \Lambda$ and $\mathcal{D E} \preceq \Lambda$.

(e) $\mathcal{E} \mathcal{D E} \simeq \mathcal{E}$ and $\mathcal{D E D} \simeq \mathcal{D}$.

Other results for morphological operators over complete lattices can also be suitably modified to yield morphological operators over complete quasi-lattices. We next consider the question: What are the suitable types of mappings? To arrive at a reasonably general answer 


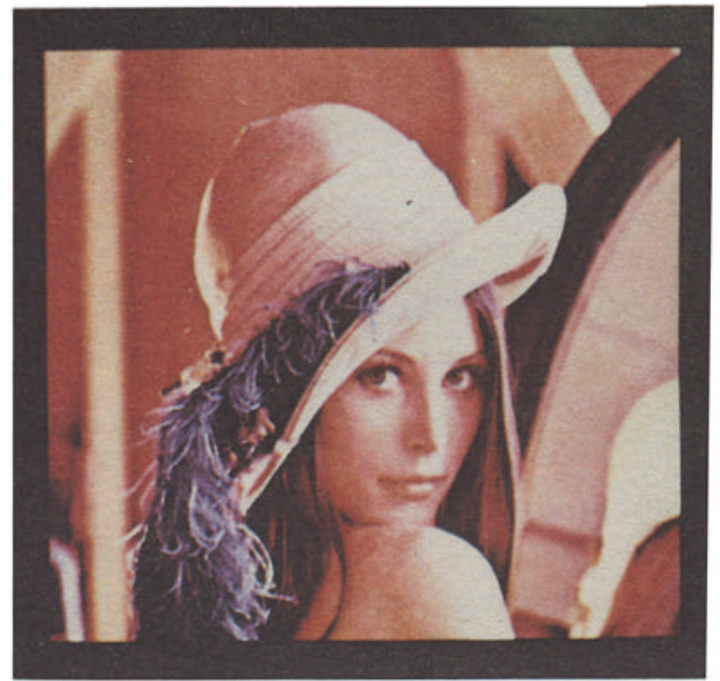

Figure 1. Colour image of Lenna $(512 \times 480)$.

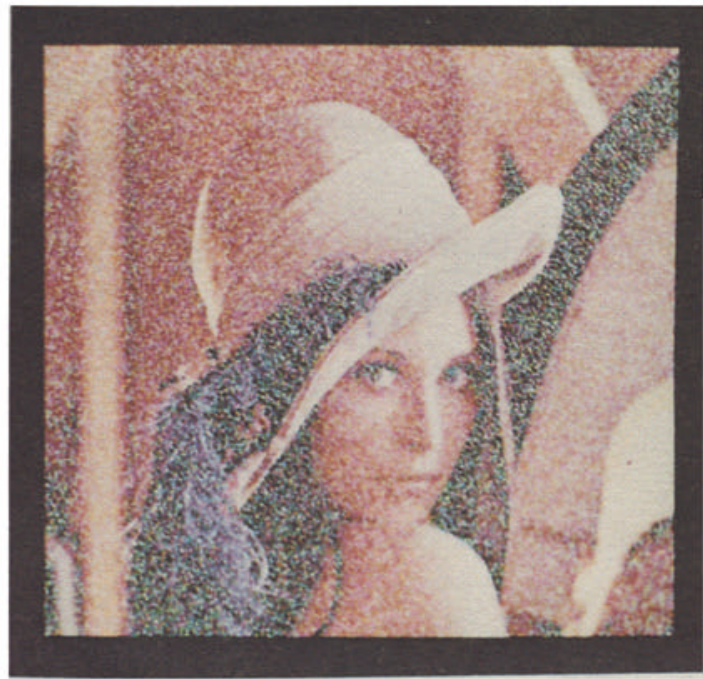

Figure 2. Image corrupted with $\operatorname{Max}$ noise with $p=0.5$.

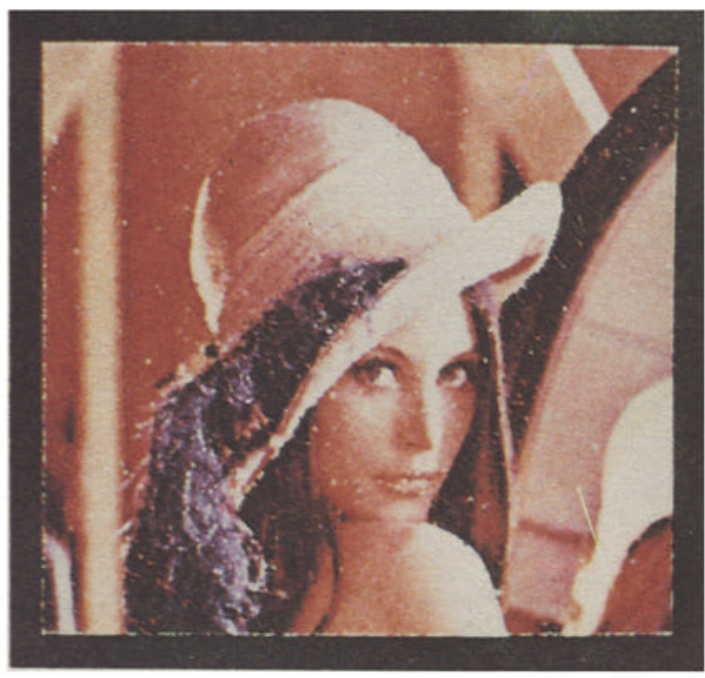

Figure 3. Image filtered using quasiopening operator.

to this question, we take recourse to Measurement Theory (Roberts 1979). We present a generalized utility function often used for conjoint measurement. 
Suppose that we have a cartesian product set $\mathcal{L}$ with a binary relation $\preceq$. Here $\mathcal{L}=$ $\prod_{i \in \mathcal{I}} \mathcal{L}_{i}$. We are in search of an ordinal utility function $\mathcal{Q}: \mathcal{L} \mapsto \mathbb{R}$. For this, we assume that there exist functions $q_{i}: \mathcal{L}_{i} \mapsto \overline{\mathbb{B}}$ for all $i \in \mathcal{I}$ and a function $q: \prod_{i \in \mathcal{I}} q_{i}\left(\mathcal{L}_{i}\right) \mapsto \overline{\mathbb{R}}$, i.e., $q: \overline{\mathbb{R}}^{m} \mapsto \overline{\mathbb{R}}$. This function $q$ is called a composition rule. Let $F \in \mathcal{L}$, then

$$
\begin{aligned}
\mathcal{Q}(F) & =\mathcal{Q}\left(f_{1}, f_{2}, \ldots, f_{m}\right) \\
& =q\left(q_{1}\left(f_{1}\right), q_{2}\left(f_{2}\right), \ldots, q_{m}\left(f_{m}\right)\right) .
\end{aligned}
$$

The composition rules that have been of most interest in the measurement literature are those where $q$ is a polynomial. Let $a \in \overline{\mathbb{R}}^{m}$, then

$$
q(a)=q\left(a_{1}, a_{2}, \ldots, a_{m}\right)=\sum_{\ell} \alpha_{\ell}\left(\prod_{i \in \mathcal{I}} a_{i}^{\beta_{i, \ell}}\right)
$$

where $\alpha_{\ell}$ are real numbers and $\beta_{i, \ell}$ are non negative integers. The specific cases of this function lead us to Euclidean and generalized distance measures used in reduced ordering (Barnett 1976) and valuations used in quasi-metric lattices (Birkhoff 1973).

Lastly, we illustrate the process of morphological filtering on a colour image. Figure 1 shows a $512 \times 480$ colour image of Lenna. Each pixel of the image has three components corresponding to the red, green and blue colour channels. This image corrupted with the Max noise is shown in figure 2. In the Max noise model, each pixel is corrupted with noise with a probability $p$ (here we have taken $p=0.5$ ), where the corrupting noise for each channel is an independent realization of a sequence of uniformly distributed random variables lying between $(0,255)$. The noise corrupts the signal by taking the maximum of the image and the noise sequence. Figure 3 shows the result of applying a $3 \times 3$ flat structuring element for the quasi-opening operator utilizing the Mahalanobis distance. It can be seen that even with such high probability of noise occurrence, the morphological filter has been able to significantly remove the corrupting noise. Similarly, for the Min noise model, a quasi-closing operator can be used to filter the multichannel image.

\section{Conclusions}

The objective of this paper is to provide a lattice theoretical framework for multivariate morphology. Within this framework, marginal ordering and reduced ordering schemes have been used to develop techniques for morphological processing of multichannel images. It has been shown that marginal ordering principle essentially leads to the matrix morphology approach. Further, it has been shown that concepts of quasi-ordering need to be utilized to develop a theory of multivariate morphology based on the reduced ordering scheme. A new concept of complete quasi-lattice is introduced which results in quasi-increasing operators, quasi-erosion/dilation operators, quasi-adjunctions etc. It remains to be seen that what kind of operators are suitable for a particular application at hand. This needs a detailed investigation to be carried out to bring out the advantages and disadvantages of various schemes for specific multichannel image processing and analysis applications.

\section{References}

Astola J, Haavisto P, Neuvo Y 1990 Vector median filters. Proc. IEEE 78: 678-689

Barnett V 1976 The ordering of multivariate data. J. R. Stat. Soc. A-139: 318-354

Birkhoff G 1973 Lattice theory. Vol. 25. American Mathematical Society Colloquim Publications

(Providence, RI) 
Cheng F, Venetsanopoulos A N 1992 Feature oriented image sequence processing and 3-D adaptive morphology - Formulation and properties. IEEE Int. Symp. Circuits Systems pp 2633-2636

Eo J W 1992 Multivariate morphological filtering with applications to colour image processing. $\mathrm{Ph} \mathrm{D}$ thesis, Oregon State University

Fuchs L 1963 Partially ordered algebraic systems (London: Pergamon)

Gierz G, Hofmann K H, Keimel K, Lawson J D, Mislove M, Scott D S 1980 A compendium of continuous lattices (New York: Springer Verlag)

Goutsias J, Sivakumar K, Heijmans H J A M 1994 Morphological operators for image sequences. Technical Report: JHU/ECE 94-09, The John Hopkins University, Baltimore

Hardie R C, Arce G R 1991 Ranking in $R^{p}$ and its use in multivariate image estimation. IEEE Trans. Circuits Syst. Video Technol. 1: 197-209

Heijmans H J A M 1994 Morphological image operators (Boston: Academic Press)

Heijmans H J A M, Ronse C 1990 The algebraic basis of mathematical morphology - Part I: Dilations and erosions. Comput. Vision, Graph. Image Process. 50: 245-295

Hseuh Y C 1992 Mathematical morphology on l-images. Signal Process. 26: 221-241

Matheron G 1975 Random sets and integral geometry (New York: Wiley)

Pitas I, Tsakalides P 1991 Multivariate ordering in color image filtering. IEEE Trans. Circuits Syst. Video Technol. 1: 247-259

Roberts F S 1979 Measurement theory with applications to decision making, utility and the social sciences. (Reading, MA: Addison-Wesley)

Ronse C 1990 Why mathematical morphology needs complete lattices. Signal Process. 21: 129154

Ronse C, Heijmans H J A M 1991 The algebraic basis of mathematical morphology - Part II: Openings and closings. Comput. Vision, Graph. Image Process.: Image Understanding 54: 74-97

Serra J 1982 Image analysis and mathematical morphology (London: Academic Press)

Serra J 1988 Image analysis and mathematical morphology, Vol.2: Theoretical advances (London: Academic Press)

Singh B, Siddiqi M U 1994 A lattice theoretical basis for mathematical multivariate morphology. Technical Report, Dept. of Electrical Eng., Indian Institute of Technology, Kanpur

Singh B, Siddiqi M U 1995 Algebraic properties of mathematical morphology on $l$-images. $J$. Math. Imaging Vision (submitted)

Stemberg S R 1986 Grayscale morphology. Comput. Vision, Graph. Image Process. 35: 333-355

Trahanias P E, Venetsanopoulos A N 1993 Color edge detection using vector order statistics. IEEE Trans. Image Process. 2: 259-264

Wilson S S 1992 Theory of matrix morphology. IEEE Trans. Pattern Anal. Machine Intell. 14: 636-652 